\title{
Therapeutic Efficacy and Inhibitory Mechanism of Regorafenib Combined With Radiation in Colorectal Cancer
}

\author{
YU-CHANG LIU ${ }^{1,2,3}$, I-TSANG CHIANG ${ }^{1,2,3}$, JING-GUNG CHUNG $^{4}$, \\ JUNG-HUNG HSIEH $^{5}$, CHIH-HUNG CHIANG ${ }^{5,6,7}$, MAO-CHI WENG $^{8}$, \\ FEI-TING HSU ${ }^{4 *}$, YUAN-HAO LEE ${ }^{*}$, CHENG-SHYONG CHANG $^{10^{*}}$, SONG-SHEI LIN $^{3 *}$ \\ ${ }^{1}$ Department of Radiation Oncology, Chang Bing Show Chwan Memorial Hospital, Changhua, Taiwan, R.O.C.; \\ ${ }^{2}$ Department of Radiation Oncology, Show Chwan Memorial Hospital, Changhua, Taiwan, R.O.C.; \\ ${ }^{3}$ Department of Medical Imaging and Radiological Sciences, \\ Central Taiwan University of Science and Technology, Taichung, Taiwan, R.O.C.; \\ ${ }^{4}$ Department of Biological Science and Technology, China Medical University, Taichung, Taiwan, R.O.C.; \\ ${ }^{5}$ Department of Urology, Medical Research and Education, \\ Taipei Veterans General Hospital, Yuan-Shan/Su-Ao Branch, Yilan, Taiwan, R.O.C.; \\ ${ }^{6}$ Department of Nursing, Cardinal Tien Junior College of Healthcare and Management, New Taipei, Taiwan, R.O.C.; \\ ${ }^{7}$ Department of Urology, National Taiwan University Hospital, Taipei, Taiwan, R.O.C.; \\ ${ }^{8}$ Isotope Application Division, Institute of Nuclear Energy Research, Taoyuan, Taiwan, R.O.C.; \\ ${ }^{9}$ Department of Ophthalmology and Visual Science, \\ University of Texas Health Science Center at Houston, Houston, TX, U.S.A.; \\ ${ }^{10}$ Division of Hematology-Oncology, Department of Internal Medicine, \\ Chang Bing Show Chwan Memorial Hospital, Changhua, Taiwan, R.O.C.
}

\begin{abstract}
Background: Although both chemotherapy and radiotherapy $(R T)$ can sufficiently maintain tumor suppression of colorectal cancer $(C R C)$, these treatments may trigger the expression of nuclear factor kappa $B(N F-$ $\left.{ }_{k} B\right)$ and compromise patients' survival. Regorafenib suppresses $N F-k B$ activity in various tumor types. However, whether regorafenib may act as a suitable radiosensitizer to
\end{abstract}

This article is freely accessible online.

*These Authors contributed equally to this study.

Correspondence to: Song-Shei Lin, Department of Medical Imaging and Radiological Sciences, Central Taiwan University of Science and Technology, Taichung 406, Taiwan, R.O.C. Tel: +886 422391647 ext. 7111, e-mail: sslin@ctust.edu.tw; Cheng-Shyong Chang, Division of Hematology-Oncology, Department of Internal Medicine, Chang Bing Show Chwan Memorial Hospital, Changhua 505, Taiwan. Tel: +886 975617091, e-mail: 1959johnchang@gmail.com; Yuan-Hao Lee, Department of Ophthalmology and Visual Science, University of Texas Health Science Center at Houston, Houston, TX 77030, U.S.A. Tel: +1 7135005988, e-mail: YUAN-HAO.LEE@uth.tmc.edu and Fei-Ting Hsu, Department of Biological Science and Technology, China Medical University, Taichung 404, Taiwan, R.O.C. Tel: +886 422053366, ext. 2532, Fax: +886422053764, e-mail: sakiro920@mail.cmu.edu.tw

Key Words: Radiotherapy, radiosensitizing effect, apoptosis, NF-kB. enhance therapeutic efficacy of $R T$ remains unknown. Materials and Methods: Here, we established a CRC-bearing animal model to investigate the therapeutic efficacy of regorafenib in combination with $R T$, through measurement of tumor growth, body weight, whole-body computed tomography (CT) scan and immunohisto-chemistry staining. Results: Smallest tumor size and weight were found in the combination treatment group. In addition, RT-induced upregulation of $N F-k B$ and downstream proteins were diminished by regorafenib. Moreover, the body weight and liver pathology in the treated group were similar to those of the non-treated control group. Conclusion: Regorafenib may enhance the anti-CRC efficacy of RT.

Recent innovative radiotherapy (RT) technologies, including intensity-modulated RT, image-guided RT, volumetric-modulated arc therapy, intraoperative RT, and stereotactic body RT (SBRT), are widely used to treat many types of cancer (1-3). RT as neoadjuvant strategy offers therapeutic benefits for patients with colorectal cancer (CRC). The combination of RT and surgical resection or chemotherapy has been shown to reduce the local recurrence rate and improve the survival of patients with CRC $(4,5)$. For increasing anti-CRC efficacy of RT, novel radiosensitizers that sensitize cancer cells to radiation have been developed (6). 
A

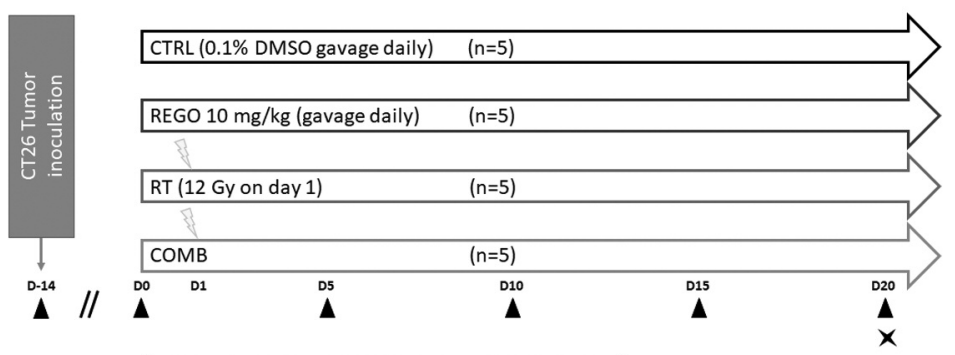

$\Delta$ Tumor volume and body weight measurement

Irradiated $12 \mathrm{~Gy}$ by linear accelerator

$X$ Whole-body CT scan and sacrificed
B

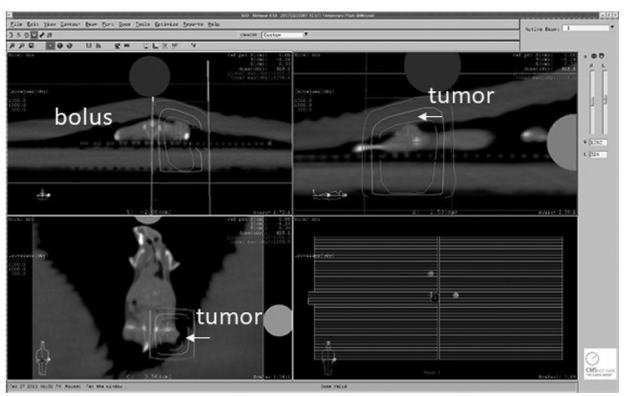

c

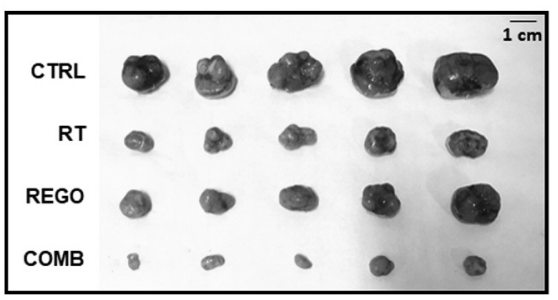

E

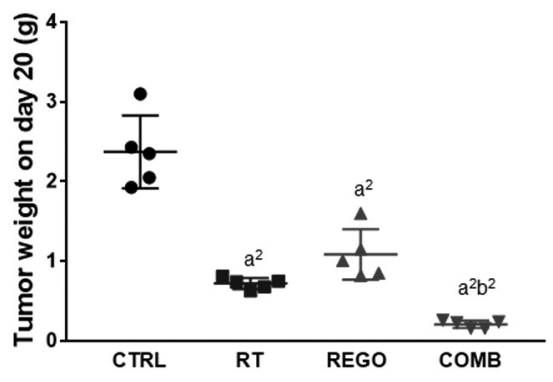

D

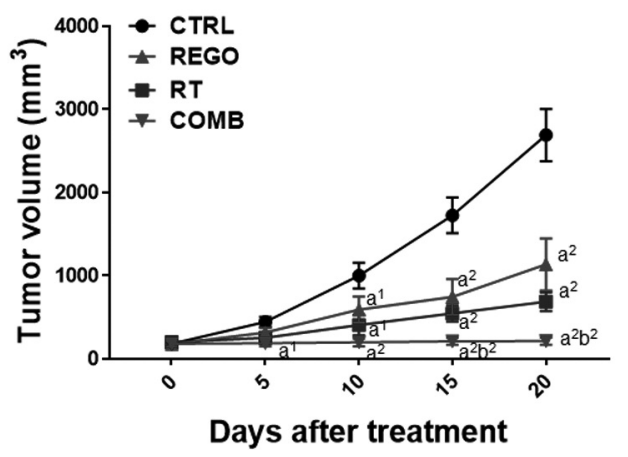

$\mathbf{F}$

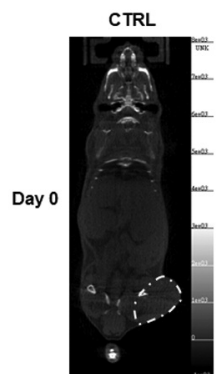

CTRL
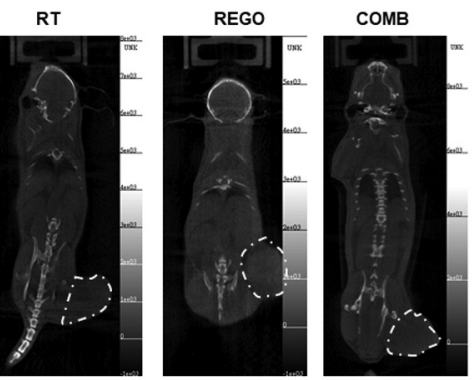

REGO

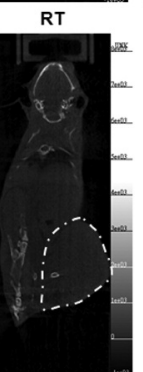

сомв

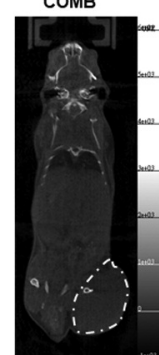

Figure 1. Regorafenib (REGO) enhanced radiotherapy (RT) inhibition of tumor growth in colorectal cancer-bearing mice. A: Experimental flow chart of animal experiment. B: Irradiation dosage and area definition are analyzed by XiO ${ }^{\circledR}$ treatment planning software. C: Photo of extracted tumors from each group of mice on day 20. D: Tumor size. E: Tumor weight. F: Whole-body computed tomographic (CT) scan images. CTRL: Control, $0.1 \%$ dimethyl sulfoxide; COMB: combination therapy. Significantly different at: $a^{1}: p<0.05$, and $a^{2}: p<0.01$ vs. control group; $b^{2}: p<0.01$ vs. single treatment group. 
A

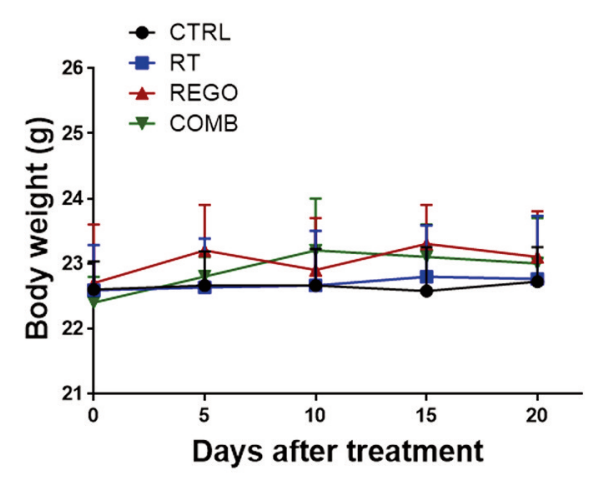

B

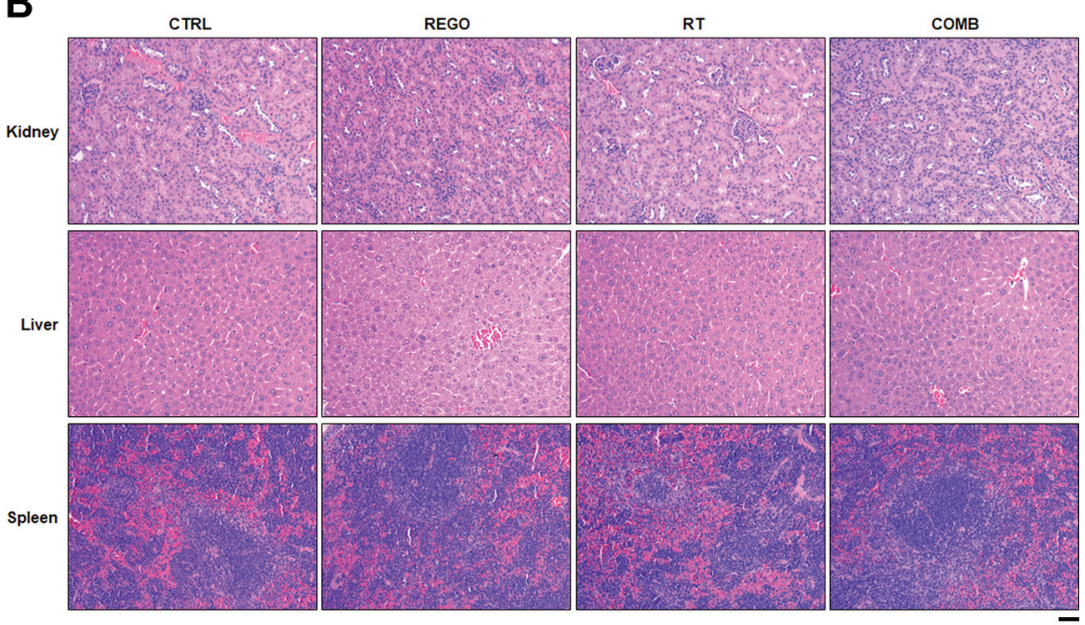

Figure 2. No general toxicity is found in mice treated with regorafenib (REGO) combined with radiotherapy (RT). A: Mouse body weight is measured every 5 days. B: Kidney, liver and spleen tissues from each group of mice on day 20 are displayed. CTRL: Control, $0.1 \%$ dimethyl sulfoxide; COMB: combination therapy. Scale bar: $100 \mu \mathrm{m}$.

Regorafenib, a Food and Drug Administration-approved oral multi-kinase inhibitor, as well as an analog of sorafenib, has been demonstrated to prolong the overall survival of patients with CRC. Regorafenib induces tumor regression through inactivation of angiogenic and oncogenic kinases such as vascular endothelial growth factor receptors 1-3, fibroblast growth factor receptor 1, epidermal growth factor receptor, RAF, tyrosine-protein kinase, and rearranged during transfection (7-10). The combination of regorafenib and SBRT was demonstrated to increase survival benefits in patient with metastatic CRC (11). However, the anti-CRC effect and mechanism of regorafenib when used along with RT is not fully understood. To harness the beneficial properties of regorafenib on CRC radiotherapy, in vivo investigative experiments were carried out to better understand its feasibility in radiosensitization and the underlying mechanism involved.

\section{Materials and Methods}

Cells, antibodies and reagents. Human CRC CT26 cells were purchased from the Bioresource Collection and Research Center (BCRC, Hsinchu, Taiwan, ROC). Cells were cultured in RPMI-1640 medium with additional $10 \%$ fetal bovine serum, $10 \mathrm{U} / \mathrm{ml}$ of penicillin and $100 \mathrm{mg} / \mathrm{ml}$ streptomycin sulfate at $37^{\circ} \mathrm{C}$ under a humidified atmosphere with $5 \% \mathrm{CO}_{2}$. Primary antibodies purchased from different companies as follow, Ki-67 (Elabscience, Houston, TX, USA), cyclin D1 (Elabscience), survivin, NF-kB p65 (Ser276) (Cell Signaling Technology, Danvers, MA, USA), cleaved caspase3 (Elabscience), cleaved caspase-8 (Elabscience), cleaved caspase9 (Proteintech, Rosemont, IL, USA), Fas cell surface death receptor (FAS) (Elabscience), Fas ligand (FAS-L) (Elabscience), myeloid cell leukemia-1 (MCL1) (Elabscience), X-linked inhibitor of apoptosis protein (XIAP) (Elabscience), and cellular FADD-like interleukin$1 \beta$-converting enzyme-inhibitory protein (C-FLIP) (Elabscience). Regorafenib and dimethyl sulfoxide (DMSO) were purchased from Sigma (St. Louis, MO, USA).

Animal experiment. Male BALB/c and CAnN.Cg-Foxn1nu/CrlNarl mice, approximately 22-28 g in weight and 4-6 weeks of age, were obtained from the National Laboratory Animal Center (Taipei, Taiwan, ROC). CT26 cells $\left(5 \times 10^{6}\right.$ cells/in $0.1-\mathrm{ml}$ phosphatebuffered saline/mouse) were subcutaneously injected into the right flank of mice and developed into CRC in 2 weeks. A total of 20 mice were randomly divided into four groups $(n=5$ each) when tumor reached $100-120 \mathrm{~mm}^{3}$ and subsequently received different treatments: Control mice were treated with $0.1 \%$ DMSO in $100 \mu \mathrm{l}$ double distilled water; regorafenib-treated mice were gavage-treated with $10 \mathrm{mg} / \mathrm{kg} /$ day regorafenib in $100 \mu \mathrm{l}$ double distilled water; RTtreated mice were treated with $12-G y$ radiation once on day 1 by linear accelerator; and the mice of the combination treatment group were treated with both regorafenib and radiation as described above (Figure 1A). Ethical approval was provided by the Institutional Animal Care and Use Committee of China Medical University (CMUIACUC-2019-288). Tumor volume was recorded every 5 days, evaluated by caliper and calculated by the equation, tumor volume=length $\times$ width ${ }^{2} \times 0.523$. The mice were sacrificed with $\mathrm{CO}_{2}$ for further studies on day 20 post-treatment.

Tumor, spleen, kidney and liver tissue collection. Tumor, spleen, kidney and liver samples were isolated from all mice after sacrificed and prepared for further processing of formalin fixing and paraffin embedding. Tumor tissues were then weighed individually.

Radiation exposure. Mice were anesthetized by 1-3\% isoflurane during the irradiation procedure. For dose build up, the tumor was covered with $1.0 \mathrm{~cm}$ bolus and scanned with computed tomography (CT). After CT simulation, $\mathrm{XiO}^{\circledR}$ treatment planning software was used to evaluate the dose distribution in the mouse. The prescribed dose given by a 

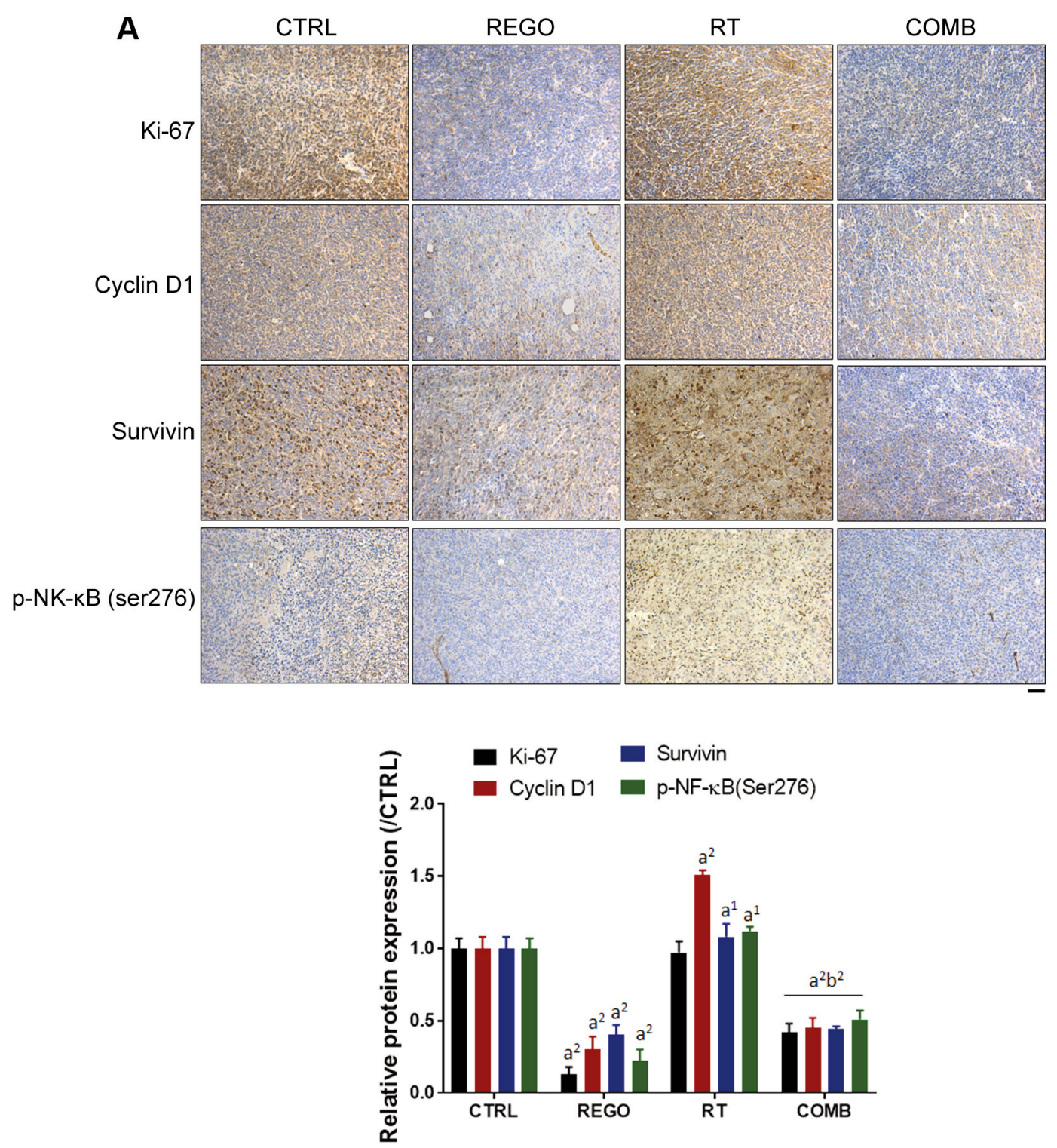

Figure 3. Continued

linear accelerator (6 MV photons; Elekta Synergy linear accelerator, Crawley, UK) to the gross tumor volume was 12 Gy (12).

Whole-body CT scan. One of the mice from each group was anesthetized by $1-3 \%$ isoflurane during whole-body CT scan on day 0 and 20. Whole-body CT scan was acquired by Mediso CT (Mediso Ltd., Budapest, Hungary). The scanning resolution was $145 \times 145 \times 145 \mu \mathrm{m}$, the tube potential peak was $55 \mathrm{kVp}$ and the tube current was $145 \mu \mathrm{A}(13)$.

Immunohistochemistry (IHC) staining. The protein expression level in tumor tissue including, Ki-67, cyclin D1, survivin, NF-kB p65 (Ser536), cleaved caspase-3, cleaved caspase-8, cleaved caspase-9, FAS, FAS-L, MCL1, XIAP, and C-FLIP were investigated in tumor tissue sections by IHC staining as described in previous studies $(9$, 14). Stained slides were imaged under a Nikon microscope at $\times 100$ (Nikon ECLIPSE Ti-U; Nikon, Minato City, Japan). Protein expression levels were measured by ImageJ software version 1.50 (National Institutes of Health, Bethesda, MD, USA).

Hematoxylin and eosin $(H \& E)$ staining. Tissue sections underwent H\&E staining by by Bio-Check Laboratories Ltd (New Taipei City, Taiwan, ROC) as described elsewhere (9). H\&E-stained sections were then imaged under microscopy and quantified by ImageJ software version 1.50 .

Statistical analysis. Data are presented as the mean \pm standard deviation $(n=5)$. All data were analyzed using GraphPad Prism 

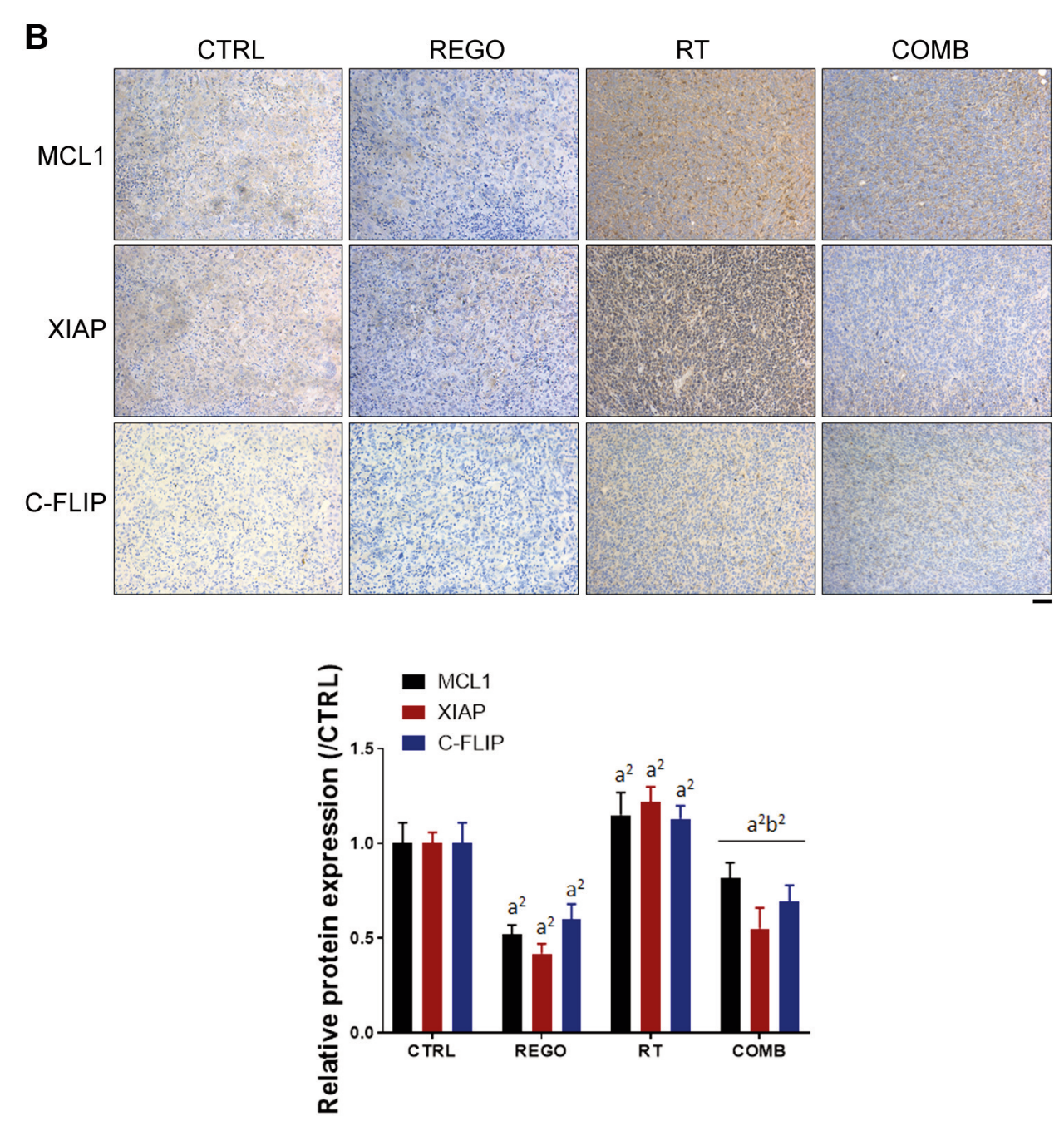

Figure 3. Regorafenib (REGO) reduced radiation (RT)-triggered nuclear factor kappa $B(N F-k B)$ activation and expression of anti-apoptosis proteins. Tumor immunostaining for expression of A: Ki-67, cyclin D1, survivin, phospho ( $p)-N F-k B($ ser276) and B: myeloid cell leukemia-1 (MCL1), $X$-linked inhibitor of apoptosis protein (XIAP), and cellular FADD-like interleukin-1 $\beta$-converting enzyme-inhibitory protein $(C$-FLIP) from each group of mice are displayed (left) and quantified (right). CTRL: Control, $0.1 \%$ dimethyl sulfoxide; COMB: combination therapy. Significantly different at: $a^{1}: p<0.05$, and $a^{2}: p<0.01 \mathrm{vs}$. control group; $b^{2}: p<0.01 \mathrm{vs}$. single treatment group. Scale bar: $100 \mu \mathrm{m}$.

version 7. Differences between the control and experimental groups were investigated by independent Student's $t$-test analysis when $p<0.05$ was considered as a statistically significant difference.

\section{Results}

Regorafenib effectively induced anti-CRC ability of RT. To investigate whether regorafenib may trigger toxicity of RT, we established CT26 tumor-bearing animal model. As shown in Figure 1A, mice were divided into four different groups: Control treated with $0.1 \%$ DMSO; RT alone (12 Gy/once); regorafenib alone $(10 \mathrm{mg} / \mathrm{kg} / \mathrm{day})$; and combination treatment. RT treatment plan was analyzed $\mathrm{XiO}^{\circledR}$ treatment planning software and irradiation was delivered by linear accelerator (Figure 1B). A 1-cm bolus for dose build up was placed on mouse tumors during irradiated procedure. Isolated tumors from each group at the endpoint (day 20) were photographed and displayed in Figure 1C. Smallest tumor size was found in the combination treatment group. Disparity in tumor volume between the combination treatment and control groups began at day 5 after treatment. After 15 days of treatment, the treatment efficacy of combination treatment group was better than that of the single treatment group (Figure 1D). Tumors extracted from mice on day 20 were weighed and the tumor weight of the combination treatment group was less than that of single treatment groups (Figure 1E). Whole-body CT scan 
indicated marked tumor growth inhibition in the combination treatment group as compared to single treatment groups (Figure 1F). In summary, as found from the tumor growth pattern, regorafenib effectively sensitized CRC to RT.

Regorafenib combined with $R T$ in CRC-bearing mice appeared not to induce general toxicity. To investigate whether regorafenib combined with RT influenced body weight alteration, we measured mouse body weight once every 5 days. As shown in Figure 2A, no significant difference change of body weight was found between untreated and treated groups. In addition, we also extracted liver, spleen, and kidney tissues from each group for pathology evaluation (Figure 2B). No noticeable pathological change was found in H\&E staining between untreated and treated groups. Taken together, regorafenib combined with RT appeared not to induce any general toxicity effect.

Regorafenib diminished radiation-induced $N F-k B$ and $N F-k B$ mediated protein expression on $C R C$-bearing mice. $\mathrm{NF}-\mathrm{kB}$ phosphorylation and the expression of NF-kB-related proteins were detected by IHC staining of tumor sections. In Figure 3A, radiation-induced phosphorylation of NF-kB was reduced by combination with regorafenib. Tumor proliferation marker, Ki67, was markedly reduced by regorafenib combined with RT. NF-kB-related proteins that regulate tumor proliferation, such as cyclin D1 and survivin were all reduced by combination therapy. Furthermore, NF-kB-related proteins that regulate apoptosis $(12,15)$, such as MCL1, XIAP and C-FLIP were also effectively reduced by regorafenib combined with RT (Figure 3B). Regorafenib successfully diminished RT-induced expression of NF-kB and its associated proteins.

Regorafenib enhanced RT-induced expression of extrinsic and intrinsic apoptosis-associated proteins in CRC-bearing mice. The levels of extrinsic and intrinsic apoptosis-related proteins, cleaved caspase-3, -8 and -9 , were detected by IHC staining of tumor sections. As illustrated in Figure 4A, overall apoptosisrelated marker cleaved caspase- 3 was significantly expressed in the combination treatment group as compared to single treatment groups. Expression of FAS, FAS-L, and cleaved caspase-8, which are extrinsic apoptosis-related proteins, were induced by regorafenib combined with RT (Figure 4B). Moreover, cleavage of caspase-9, an intrinsic apoptosis-related protein was also triggered in the combination treatment group as compared to single treatment group. In conclusion, RTinduced expression of extrinsic and intrinsic apoptotic-related proteins were successfully enhanced by regorafenib.

\section{Discussion}

The combination of regorafenib and SBRT has been demonstrated to control disease progression and extend progression-free survival to approximately 3 years in patients with late-stage metastatic CRC (11). Our results indicate that regorafenib, RT, and their combination significantly diminished tumor growth of CT-26 tumor-bearing mice (Figure 1C and D). The combination group exhibited small tumor volume and tumor weight compared to mice from individual single treatment groups (Figure 1E). We found that regorafenib, as a radiosensitizer, effectively diminished CRC tumor growth with RT in vivo.

Cleaved caspase- 8 and -9 are required for apoptosis induced by radiation through extrinsic (via death receptor) and intrinsic (mitochondrial) apoptotic pathways. Caspase-3 can be activated or cleaved by caspase- 8 or caspase- 9 , leading to initiation of apoptotic DNA fragmentation (16, 17). The increased protein expression of cleaved caspase- 3 in tumor and tumor-associated stroma was associated with better prognosis in patients with CRC (18). Our results showed that expression of FAS, FAS-L, and cleaved caspase$3,-8$, and -9 was significantly augmented with regorafenib, RT, and their combination (Figure 4). The combination treatment increased the expression of these proteins to a significantly higher level than that increased by regorafenib or radiation alone.

$\mathrm{NF}-\mathrm{kB}$, an oncogenic transcription factor composed of p50 and p65 dimers, can be activated with chemotherapeutic agents or radiation $(19,20)$. Increased NF-kB activity attenuates the anticancer efficacy of RT through promoting the expression of proliferative and anti-apoptotic proteins, such as cyclin-D1, survivin, MCL1, XIAP, and C-FLIP (12, $21)$. It has been found that worse 3- and 5-year overall survival of patients with CRC was correlated with overexpression of NF-kB activation (22). In addition, cell and animal models revealed suppression of $\mathrm{NF}-\mathrm{kB}$ signaling enhanced radiosensitivity of $\mathrm{CRC}(12,15,21)$. Correspondingly, we found that radiation-triggered NF-kB activation, expression of proliferative, and anti-apoptotic proteins were significantly diminished by regorafenib concurrent treatment (Figure 3).

In conclusion, regorafenib was found to enhance the anti$\mathrm{CRC}$ efficacy of RT. Induction of apoptosis and reduction of $\mathrm{NF}-\mathrm{kB}$ signaling are involved in the radiosensitizing effect of regorafenib.

\section{Conflicts of Interest}

The Authors declare no competing financial interests regarding this study.

\section{Authors' Contributions}

YCL, JHH, MCW and FTH performed the experiments. YHL, ITC and CSC prepared the initial version of the article. YCL, JGC and SSL designed the study, performed the literature review, and prepared the final versions of the article. 


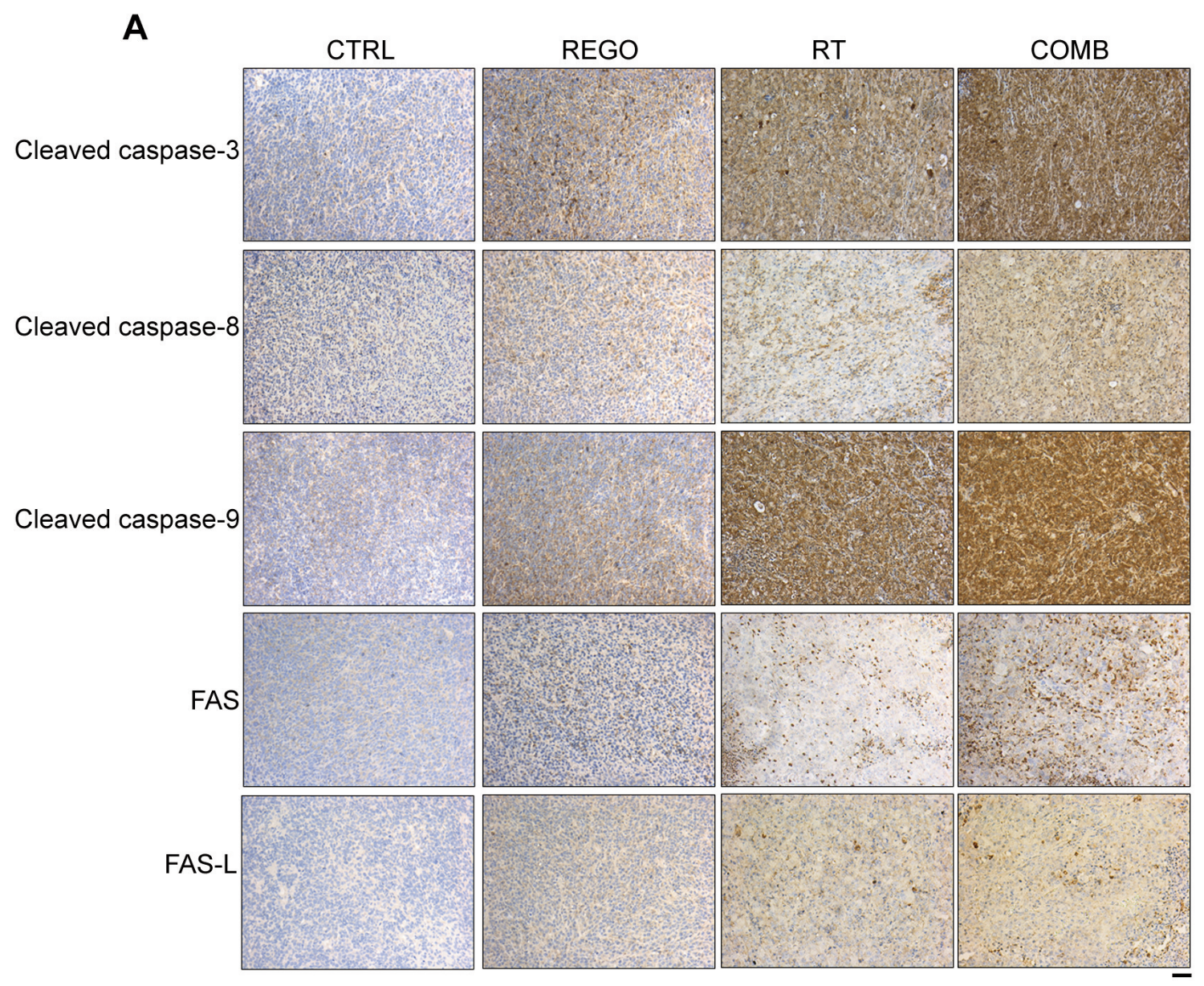

B

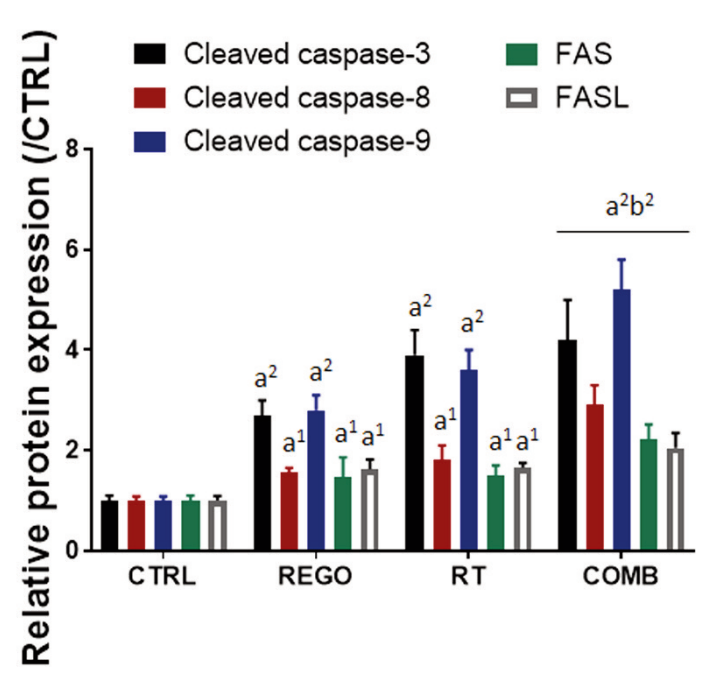

Figure 4. Regorafenib (REGO) increased expression of radiation (RT)-induced apoptotic proteins. A: Immunohistochemistry staining images and B: quantification of cleaved caspase-3, -8 and -9, Fas cell surface death receptor (FAS), and Fas ligand (FAS-L) expression in tumor from each group of mice are displayed. CTRL: Control, $0.1 \%$ dimethyl sulfoxide; COMB: combination therapy. Significantly different at: $a^{1}: p<0.05$, and $a^{2}$ : $p<0.01$ vs. control group $\left(0.1 \%\right.$ dimethyl sulfoxide; CTRL); $b^{2}: p<0.01$ vs. single treatment group. Scale bar: $100 \mu \mathrm{m}$. 


\section{Acknowledgements}

This study were supported by Chang Bing Show Chwan Memorial Hospital (ID: BRD-18010), Taipei Veterans General Hospital, YuanShan/Su-Ao Branch, Yilan (ID: YSVH10904) and Central Taiwan University of Science and Technology (ID: CTU108-P-103), respectively.

\section{References}

1 Fiorino C, Guckemberger M, Schwarz M, van der Heide UA and Heijmen B: Technology-driven research for radiotherapy innovation. Mol Oncol 14(7): 1500-1513, 2020. PMID: 32124546. DOI: 10.1002/1878-0261.12659

2 Wu C-H, Chen C-Y, Yeh C-T and Lin K-H: Radiosensitization of hepatocellular carcinoma through targeting radio-associated microRNA. Int J Mol Sci 21(5): 1859, 2020. PMID: 32182776. DOI: $10.3390 /$ ijms 21051859

3 Tam SY and Wu VWC: A review on the special radiotherapy techniques of colorectal cancer. Front Oncol 9: 208, 2019. PMID: 31001474. DOI: 10.3389/fonc.2019.00208

4 Liu H, Zhang L, Li G and Gao Z: Xanthohumol protects against azoxymethane-induced colorectal cancer in Sprague-Dawley rats. Environ Toxicol 35(2): 136-144, 2020. PMID: 31714664. DOI: $10.1002 /$ tox. 22849

5 Kim JH: Controversial issues in radiotherapy for rectal cancer: A systematic review. Radiat Oncol J 35(4): 295-305, 2017. PMID: 29325395. DOI: 10.3857/roj.2017.00395

6 Calvaruso M, Pucci G, Musso R, Bravatà V, Cammarata FP, Russo G, Forte GI and Minafra L: Nutraceutical compounds as sensitizers for cancer treatment in radiation therapy. Int J Mol Sci 20(21): 5267, 2019. PMID: 31652849. DOI: 10.3390/ijms 20215267

7 Mercier J and Voutsadakis IA: A systematic review and metaanalysis of retrospective series of regorafenib for treatment of metastatic colorectal cancer. Anticancer Res 37(11): 5925-5934, 2017. PMID: 29061771. DOI: 10.21873/anticanres.12039

8 Krishnamoorthy SK, Relias V, Sebastian S, Jayaraman V and Saif MW: Management of regorafenib-related toxicities: A review. Therap Adv Gastroenterol 8(5): 285-297, 2015. PMID: 26327919. DOI: $10.1177 / 1756283 X 15580743$

9 Liu YC, Tsai JJ, Weng YS and Hsu FT: Regorafenib suppresses epidermal growth factor receptor signaling-modulated progression of colorectal cancer. Biomed Pharmacother 128: 110319, 2020. PMID: 32502841. DOI: 10.1016/j.biopha.2020. 110319

10 Jensen LH, Olesen R, Petersen LN, Boysen AK, Andersen RF, Lindebjerg J, Nottelmann L, Thomsen CEB, Havelund BM, Jakobsen A and Hansen TF: NPY gene methylation as a universal, longitudinal plasma marker for evaluating the clinical benefit from last-line treatment with regorafenib in metastatic colorectal cancer. Cancers 11(11): 1649, 2019. PMID: 31731482. DOI: $10.3390 /$ cancers 11111649

11 Roberto M, Falcone R, Mazzuca F, Archibugi L, Castaldi N, Botticelli A, Osti MF and Marchetti P: The role of stereotactic body radiation therapy in oligometastatic colorectal cancer: Clinical case report of a long-responder patient treated with regorafenib beyond progression. Medicine 96(48): e9023, 2017. PMID: 29310420. DOI: 10.1097/MD.0000000000009023
12 Chen YS, Sun R, Chen WL, Yau YC, Hsu FT, Chung JG, Tsai $\mathrm{CJ}$, Hsieh $\mathrm{CL}$, Chiu $\mathrm{YM}$ and Chen JH: The in vivo radiosensitizing effect of magnolol on tumor growth of hepatocellular carcinoma. In Vivo 34(4): 1789-1796, 2020. PMID: 32606148. DOI: 10.21873/invivo.11973

13 Weng MC, Li MH, Chung JG, Liu YC, Wu JY, Hsu FT and Wang HE: Apoptosis induction and AKT/NF-kB inactivation are associated with regroafenib-inhibited tumor progression in nonsmall cell lung cancer in vitro and in vivo. Biomed Pharmacother 116: 109032, 2019. PMID: 31163381. DOI: 10.1016/j.biopha. 2019.109032

14 Su CM, Weng YS, Kuan LY, Chen JH and Hsu FT: Suppression of $\mathrm{PKC} / \mathrm{NF}-\mathrm{KB}$ signaling and apoptosis induction through extrinsic/intrinsic pathways are associated magnolol-inhibited tumor progression in colorectal cancer in vitro and in vivo. Int $\mathrm{J}$ Mol Sci 21(10): 3527, 2020. PMID: 32429376. DOI: 10.3390/ ijms 21103527

15 Sandur SK, Deorukhkar A, Pandey MK, Pabón AM, Shentu S, Guha S, Aggarwal BB and Krishnan S: Curcumin modulates the radiosensitivity of colorectal cancer cells by suppressing constitutive and inducible NF-kappaB activity. Int J Radiat Oncol Biol Phys 75(2): 534-542, 2009. PMID: 19735878. DOI: 10.1016/j.ijrobp.2009.06.034

16 Rahmanian N, Hosseinimehr SJ and Khalaj A: The paradox role of caspase cascade in ionizing radiation therapy. J Biomed Sci 23(1): 88, 2016. PMID: 27923354. DOI: 10.1186/s12929-0160306-8

17 Manente L, Pecoraro S, Picillo E, Gargiulo U, Gargiulo P, De Luca A and Politano L: Molecular evidence of apoptotic pathway activation in semen samples with high DNA fragmentation. In Vivo 29(2): 289-294, 2015. PMID: 25792659.

18 Noble P, Vyas M, Al-Attar A, Durrant S, Scholefield J and Durrant L: High levels of cleaved caspase-3 in colorectal tumour stroma predict good survival. Br J Cancer 108(10): 2097-2105, 2013. PMID: 23591201. DOI: 10.1038/bjc.2013.166

19 Celec P: Nuclear factor kappa B-molecular biomedicine: The next generation. Biomed Pharmacother 58(6-7): 365-371, 2004. PMID: 15271418. DOI: 10.1016/j.biopha.2003.12.015

$20 \mathrm{Li} \mathrm{F}$ and Sethi G: Targeting transcription factor nuclear factor kappa B to overcome chemoresistance and radioresistance in cancer therapy. Biochim Biophys Acta 1805(2): 167-180, 2010. PMID: 20079806. DOI: 10.1016/j.bbcan.2010.01.002

21 Kunnumakkara AB, Diagaradjane P, Guha S, Deorukhkar A, Shentu S, Aggarwal BB and Krishnan S: Curcumin sensitizes human colorectal cancer xenografts in nude mice to gammaradiation by targeting nuclear factor kappa B-regulated gene products. Clin Cancer Res 14(7): 2128-2136, 2008. PMID: 18381954. DOI: 10.1158/1078-0432.CCR-07-4722

$22 \mathrm{Wu}$ D, Wu P, Zhao L, Huang L, Zhang Z, Zhao S and Huang J: NF-kB expression and outcomes in solid tumors: A systematic review and meta-analysis. Medicine 94(40): e1687, 2015. PMID: 26448015. DOI: 10.1097/MD.0000000000001687

Received August 1, 2020

Revised August 24, 2020 Accepted August 26, 2020 